\title{
Early Structural Deterioration of a Sutureless Bioprosthetic Aortic Valve
}

\author{
Michael Cinelli ${ }^{\mathrm{a}, \mathrm{d}}$, Leonard Schwartz ${ }^{\mathrm{a}}$, Jonathan Spagnola ${ }^{\mathrm{b}}$, Iosif Gulkarov , \\ Frank Rosell ${ }^{c}$, Adam Lackey ${ }^{\mathrm{c}}$, Mohammed Imam ${ }^{\mathrm{c}}$, Charles Schwartz
}

\begin{abstract}
Sutureless bioprosthetic valves such as the Sorin Perceval S valve (SPV) have been used in patients with aortic stenosis that require surgical aortic valve replacement (SAVR). These prostheses have been marketed on the basis of their rapid implantation techniques with avoidance of sutures and reduced aortic cross-clamp times. We report a case of an early failure of a SPV nearly 4 years after implantation in a 58-year-old woman who was low-risk. While the patient's symptoms initially improved with SAVR with a sutureless bioprosthetic valve, they progressively worsened as the valve degraded, and the leaflets became increasingly calcified and stenotic ultimately, requiring reoperative SAVR with a St. Jude mechanical valve. This case raises the issue of the lack of much-needed data describing the long-term durability and hemodynamic performance of these valves, particularly in a low-risk patient with excellent functional status. We hope to shed further insight into the lack of long-term studies on patients with SPV to assess their longevity and long-term effectiveness, as well as elucidation of possible prevention and monitoring of these potential complications. The use of newer generation prostheses, although attractive for their ease of implantation, potentially carries higher long-term risk due to shorter durability leading to reintervention to address valve deterioration. This is especially true in low-risk patients who are young and active. Cardiology and cardiothoracic surgery societies need to develop a universal registry with follow-up of all valves in order to track and study the durability of these valves, and to evaluate for incidence of known and potential complications.
\end{abstract}

Keywords: Sorin Perceval; Aortic valve replacement; Bioprosthetic valve; Bioprosthetic valve failure; Sutureless valve failure; Surgical aortic valve replacement; Aortic stenosis; Sutureless valve

Manuscript submitted January 27, 2020, accepted February 18, 2020

aDepartment of Medicine, Staten Island University Hospital, Staten Island, NY, USA

bDivision of Cardiology, Department of Medicine, Staten Island University Hospital, Staten Island, NY, USA

'Department of Cardiothoracic Surgery, Staten Island University Hospital, Staten Island, NY, USA

${ }^{\mathrm{d} C o r r e s p o n d i n g ~ A u t h o r: ~ M i c h a e l ~ C i n e l l i, ~} 475$ Seaview Ave, Staten Island, NY 10305, USA.Email: mcinelli1@northwell.edu

doi: https://doi.org/10.14740/cr1013

\section{Introduction}

Aortic stenosis (AS) is a restriction in the movement of the aortic valve leaflets, with consequent obstruction to the flow of blood. The characteristic presenting symptoms are classically dyspnea, syncope and chest pain [1]. The gold standard treatment for symptomatic AS is surgical aortic valve replacement (SAVR) $[2,3]$. Classically there are two types of prosthetic valves used in the management of this disease: mechanical and bioprosthetic. Mechanical prostheses have the advantage of very long durability, proven over several decades and thousands of patient-years of follow-up. They however require lifelong anticoagulation to prevent thromboembolism and consequently expose the patient to bleeding complications. Bioprosthetic valves have become increasingly favorable as they do not require lifelong anticoagulation due the decreased risk of thromboembolic events compared to mechanical heart valves [4].

Sutureless bioprosthetic valves such as the Sorin Perceval valve (Sorin Biomedica Cardio Srl, Saluggia, Italy) have been used in patients with AS that required SAVR. They have been marketed on the attractive feature that it does not require sutures during implantation. It is a self-expanding bioprosthetic valve comprised of leaflets made of bovine pericardium mounted within a self-expanding, super elastic nitinol alloy stent frame $[5,6]$. As these valves are relatively new on the market, their long-term durability has yet to be fully established. We describe a failed Sorin Perceval S valve (SPV) that was replaced surgically with a St. Jude mechanical valve.

\section{Case Report}

A 58-year-old female with a past medical history of SAVR 4 years prior with SPV due to calcific AS, history of myocardial infarction status post percutaneous coronary intervention to the right coronary artery, hypertension, chronic obstructive pulmonary disease, obstructive sleep apnea, Crohn's disease, thalassemia trait, and gastroesophageal reflux presented with progressive exertional dyspnea, paroxysmal nocturnal dyspnea, and chest discomfort for the past 6 months. Her medications included amlodipine, losartan, furosemide, aspirin, clopidogrel, metoprolol succinate, atorvastatin, pantoprazole, and budesonide/formoterol. Her calcium was $8.9 \mathrm{mg} / \mathrm{dL}$ and phosphorus was $3.6 \mathrm{mg} / \mathrm{dL}$. Her creatinine was $0.8 \mathrm{mg} / \mathrm{dL}$ 


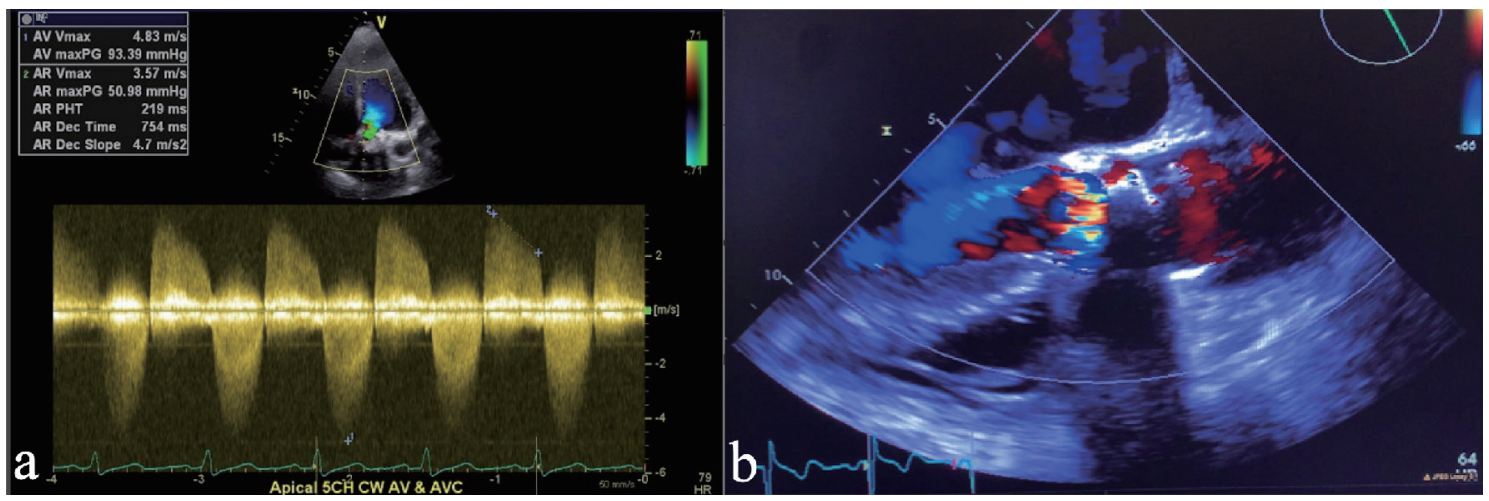

Figure 1. (a) Continuous wave (CW) Doppler on transthoracic echocardiogram showing peak aortic valve gradient of $93.39 \mathrm{~mm}$ $\mathrm{Hg}$, consistent with severe aortic stenosis. (b) Transthoracic echocardiogram showing severe calcification and aortic valve stenosis consistent with bioprosthetic aortic valve failure.

with a glomerular filtration rate of $81 \mathrm{~mL} / \mathrm{min} / 1.73 \mathrm{~m}^{2}$. B-type natriuretic peptide (BNP) was $6,960 \mathrm{pg} / \mathrm{mL}$. Her hemoglobin was $11.7 \mathrm{~g} / \mathrm{dL}$ and hematocrit was $35.6 \%$. Prothrombin time (PT) was $11.6 \mathrm{~s}$, activated partial thromboplastin time was 26.7 $\mathrm{s}$ and international normalized ratio (INR) was 1.07 , which were around her baseline. All other routine lab work including cardiac enzymes was unremarkable. Chest X-ray showed bilateral interstitial opacities. An electrocardiogram (ECG) showed normal sinus rhythm. Transthoracic echocardiogram revealed bioprosthetic AS with a peak aortic velocity of 4.83 $\mathrm{m} / \mathrm{s}$, a peak transaortic gradient of $93.39 \mathrm{~mm} \mathrm{Hg}$ (Fig. 1a), a mean transaortic gradient of $53.0 \mathrm{~mm} \mathrm{Hg}$, an aortic valve area of $0.62 \mathrm{~cm}^{2}$, mild to moderate mitral valve regurgitation, left ventricular ejection fraction of $60 \%$, and severe aortic root calcification (Fig. 1b) (Video 1, www.cardiologyres.org).

The patient was evaluated in valve clinic, and was initially considered for transcatheter aortic valve replacement (TAVR). She underwent cardiac computed tomography angiogram (CTA) and TAVR CTA to evaluate for access. The bioprosthetic aortic valve leaflets were noted to be thickened and calcified consistent with severe bioprosthetic aortic valve failure.

Valve-in-valve (ViV)-TAVR was considered by the structural heart team, but given the patient's young age, excellent functional status, and potential for requiring another aortic valve repair (AVR) in her lifetime, it was decided that SAVR with a mechanical valve was a better option. A transesophageal echocardiogram showed no evidence of infective endocarditis but demonstrated significant calcified pannus, which raised concern for risk of embolization with TAVR. The patient's Society of Thoracic Surgeons (STS) score was calculated to be $4 \%$. Cardiac catheterization showed no significant coronary artery disease.

The patient underwent a successful reoperative SAVR with a $23-\mathrm{mm}$ St. Jude mechanical aortic valve via sternotomy. Intraoperatively, the SPV was explanted and severely calcified, and stiffened leaflets were noted, consistent with the echocardiogram (Fig. 2) (Video 2, www.cardiologyres.org). The patient was started on anticoagulation with coumadin for the St. Jude mechanical valve. She felt marked relief in her symptoms to the extent of no longer requiring continuous positive airway pressure therapy for her obstructive sleep apnea.

\section{Discussion}

This is a case of a failed SPV nearly 4 years after implantation. The patient initially improved after the initial aortic valve replacement (AVR) with bioprosthetic SPV, but over time, progressively worsened as the valve degraded and the leaflets became calcified.

Failure of SPV has been described in varying short-term time frames even as early as 5 days [7] to 3 weeks [8] to 5 years after implantation [9]. Multiple mechanisms of failure of the SPV have been reported. The most common described mechanism of failure was so-called "stent creep" wherein the valve framework bends inward thereby deforming the valve leading to an increased aortic valvular gradient which ultimately leads to valve failure [10]. There are a number of reports of $\mathrm{ViV}$ TAVR repair of SPV failure due to stent creep [7-14].

The second most common described bioprosthetic valve failure, much like native valve, is calcium deposition, which clearly was demonstrated in our patient in the nodular calci-

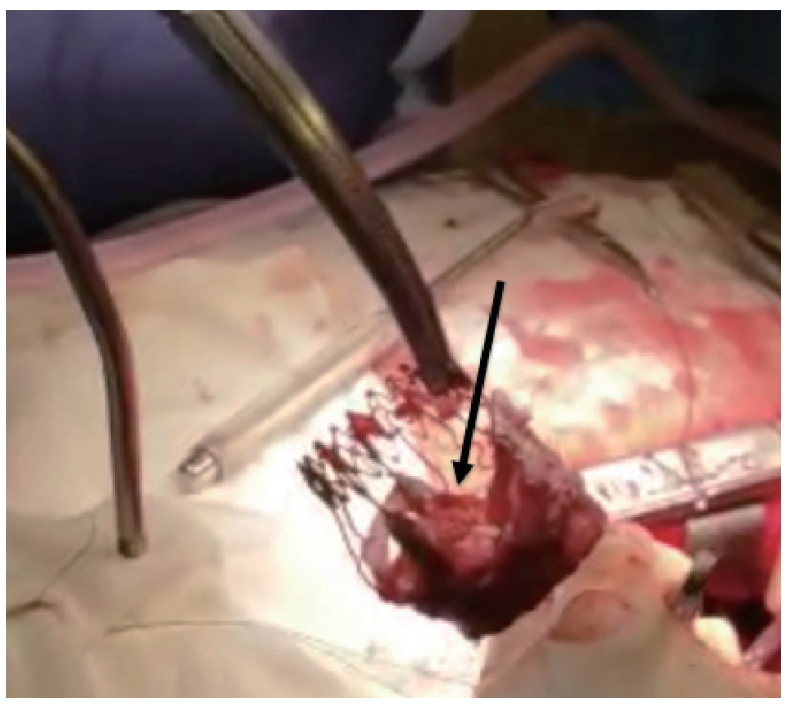

Figure 2. Surgically explanted Sorin Perceval $S$ bioprosthetic valve showing calcified leaflets (black arrow). 
fications on the valve leaflets alongside an observed pannus formation likely due to collagen degradation [10]. This constitutes a classic mechanism of failure, not only of SPV but bioprosthetic valves in general. This mimics the pathophysiology of native valvular calcification that leads to stenosis over time. To our knowledge, our patient is one of the few reported cases in addition to the report by Mangner et al [9] where an SPV undergoes structural deterioration due to valvular calcification. Mangner et al described a case of a 75-year-old woman with failed SPV at 5 years for which she underwent ViV-TAVR with a SAPIEN 3 valve.

One of the major incentives to use sutureless bioprosthetic valves like the SPV is ease of implantation that results in decreased aortic cross-clamp and cardiopulmonary bypass times, which are strongly associated with morbidity and mortality after the procedure $[6,15]$. The SPV reduces these times by as much as $50 \%$ [8] thereby making the valve very attractive to cardiothoracic surgeons. Furthermore, multiple analyses have shown that the risk of early mortality is lower in patients who received sutureless valves in comparison to sutured valves [16].

Management of failed SPV can be percutaneous or surgical. Patient age, estimated long-term survival and benefit must be considered when deciding between ViV-TAVR and SAVR. In our opinion, younger patients probably should not be considered for a valve like SPV due to the lack of robust durability data. This begs the question of whether to preferentially use these valves in patients who are older and have a more succinct timeline with that of the natural wear and tear of an SPV. Nonetheless, SAVR remains a route that proves to be very effective and long lasting for most patient populations. The complexity of valve choice for SAVR should also be considered as the untested durability of newer generation, rapid deployment or sutureless aortic valves, relative to standard techniques for time-tested prostheses with long lasting success is unmatched. While a recent meta-analysis showed that ViV-TAVR is a good proven redo option for patients with failed bioprosthetic valves whom are considered high-risk [17]; it is not as clear for a patient that is low- or even intermediate-risk.

Low-risk patients who receive bioprosthetic valves will likely require reoperative SAVR as they outlive the durability of the valves [13]. As many as $40 \%$ of bioprosthetic valves fail in younger patient populations compared to older patients [18]. Most bioprosthetic valves are expected to last 10 - 20 years but later succumb to degradation which presents as AS and potential concomitant insufficiency [14].

This case raises the issue of the lack of much-needed data describing the long-term durability and hemodynamic performance of sutureless bioprosthetic valves, particularly in a lowrisk patient with excellent functional status. The SPV was only introduced in 2008 with a total of about 2,500 valves placed to date worldwide [19]. Therefore, the data on long-term durability are scarce to none. Most of the studies reported shortterm results describing hemodynamic parameters and 30-day outcomes $[5,6,16,19,20]$. One multicenter study reported 5-year follow-up data and showed favorable hemodynamic parameters such as transaortic valve gradient as well as patient symptomatology [2].

In the TAVR era where mainly low-risk patients are re- ferred for SAVR, it is imperative to consider valve durability, as all of these low-risk patients are likely to outlive the durability of implanted valves. Valve properties that facilitate surgery and reduce clamp-time are secondary to durability of a valve in low-risk patients who are able to tolerate slightly prolonged clamp and cardiopulmonary bypass time without any side effects. It is very reasonable to offer these low-risk patients, in the absence of contraindication for anticoagulation with coumadin, mechanical bioprosthesis that have much better durability than any bioprosthetic valve.

We hope to shed further insight into the lack of longterm studies on patients with SPV to assess the longevity and long-term effectiveness of these valves. Our goal is for operators to be aware of the potential complications and lack of clearly defined evidence in long-term efficacy and durability when attempting to place one of these valves. Since the advent of the valve being used in the first patients in 2008, many reports of valve failure have been described. Only time and further experiences will define the durability of SPV. We further exemplify the much-needed data in both the durability of sutureless bioprosthetic valves as well as elucidation of possible prevention and monitoring of this potential complication [21].

Publication of robust data on the long-term durability of bioprosthetic valves is still ongoing. In the interim it appears reasonable for SAVR with a valve that has well-established long-term durability in patients with low surgical risk and long life expectancy to be more favorable and beneficial to the patient. Cardiology and cardiothoracic surgery societies need to develop a universal registry with follow-up of all valves in order to track and study the durability of these valves and evaluate for incidence of known and potential complications.

\section{Conclusions}

The use of newer generation bioprostheses, although attractive for their ease of implantation, can potentially carry higher long-term risk due to their shorter durability leading to reintervention to address valve deterioration. This is especially true in low-risk patients who are young and active. These patients are likely better served with the time-tested prostheses with long-established durability and freedom from structural deterioration. A mechanical valve in a young, otherwise healthy and compliant patient has an excellent long-term prognosis despite the risks of lifelong anticoagulation and bleeding or thrombosis. However, sutureless bioprosthetic valve durability is known to be limited, and their use in younger, active patients is controversial, even if quite attractive as an alternative choice for AVR. Additionally, technical intraoperative complications can occur as there is a relative lack of operative experience with these valves.

The type of AVR chosen should take into account comparison between the demonstrated durability of the bioprosthesis versus the life expectancy of the patient. Development of a universal registry with follow-up of all valves in order to track and study durability of these valves and evaluate for known and potential complications is much needed to address this issue. 


\section{Supplementary Material}

Video 1. Transthoracic echocardiogram showing calcified aortic prosthesis with evidence of severe aortic stenosis consistent with bioprosthetic aortic valve failure.

Video 2. Intraoperative surgically explanted Sorin Perceval S bioprosthetic valve showing severely calcified and stiffened leaflets.

\section{Acknowledgments}

None to declare.

\section{Financial Disclosure}

None to declare.

\section{Conflict of Interest}

None to declare.

\section{Informed Consent}

Obtained.

\section{Author Contributions}

CS and LS presented the idea and assisted in the design of the paper. MC and LS performed background search, designed and co-wrote the paper. MC, JS, LS, IG, FR, and CS co-wrote and revised the paper. CS, IG, MI, FR and AL provided mentorship and revision of the paper.

\section{Data Availability}

The authors declare that data supporting the findings of this study are available within the article.

\section{References}

1. Ross J, Jr., Braunwald E. Aortic stenosis. Circulation. 1968;38(1 Suppl):61-67.

2. Shrestha M, Fischlein T, Meuris B, Flameng W, Carrel T, Madonna F, Misfeld M, et al. European multicentre experience with the sutureless Perceval valve: clinical and haemodynamic outcomes up to 5 years in over 700 patients. Eur J Cardiothorac Surg. 2016;49(1):234-241.

3. Nishimura RA, Otto CM, Bonow RO, Carabello BA, Erwin JP, 3rd, Guyton RA, O'Gara PT, et al. 2014 AHA/ ACC Guideline for the Management of Patients With
Valvular Heart Disease: executive summary: a report of the American College of Cardiology/American Heart Association Task Force on Practice Guidelines. Circulation. 2014;129(23):2440-2492.

4. Maganti M, Rao V, Armstrong S, Feindel CM, Scully HE, David TE. Redo valvular surgery in elderly patients. Ann Thorac Surg. 2009;87(2):521-525.

5. Lio A, Miceli A, Solinas M, Glauber M. Initial experience with sutureless sorin perceval $\mathrm{S}$ aortic prosthesis for the treatment of prosthetic valve endocarditis. Thorac Cardiovasc Surg. 2015;63(6):501-503.

6. Fischlein T, Meuris B, Hakim-Meibodi K, Misfeld M, Carrel T, Zembala M, Gaggianesi S, et al. The sutureless aortic valve at 1 year: A large multicenter cohort study. J Thorac Cardiovasc Surg. 2016;151(6):1617-1626 e11614.

7. Di Eusanio M, Saia F, Pellicciari G, Phan K, Ferlito M, Dall'Ara G, Di Bartolomeo R, et al. In the era of the valve-in-valve: is transcatheter aortic valve implantation (TAVI) in sutureless valves feasible? Ann Cardiothorac Surg. 2015;4(2):214-217.

8. Sun X, Song Z, Soffer D, Pirris JP. Transcatheter aortic valve-in-valve implantation for early failure of sutureless aortic bioprosthesis. J Card Surg. 2018;33(4):172-175.

9. Mangner N, Holzhey D, Misfeld M, Linke A. Treatment of a degenerated sutureless Sorin Perceval(R) valve using an Edwards SAPIEN 3. Interact Cardiovasc Thorac Surg. 2018;26(2):364-366.

10. Attia R, Papalexopoulou N, Hancock J, Young C, Thomas M, Bapat V. Successful treatment of failing biological prosthesis because of "Stent creep" with valve-in-valve transcatheter aortic valve implantation. Catheter Cardiovasc Interv. 2015;86(2):E119-125.

11. Bapat V, Attia R, Redwood S, Hancock J, Wilson K, Young C, Thomas M. Use of transcatheter heart valves for a valve-in-valve implantation in patients with degenerated aortic bioprosthesis: technical considerations and results. J Thorac Cardiovasc Surg. 2012;144(6):13721379; discussion 1379-1380.

12. Dvir D, Webb JG, Bleiziffer S, Pasic M, Waksman R, Kodali S, Barbanti M, et al. Transcatheter aortic valve implantation in failed bioprosthetic surgical valves. JAMA. 2014;312(2):162-170.

13. Ferrari E, Marcucci C, Sulzer C, von Segesser LK. Which available transapical transcatheter valve fits into degenerated aortic bioprostheses? Interact Cardiovasc Thorac Surg. 2010;11(1):83-85.

14. Webb JG, Dvir D. Transcatheter aortic valve replacement for bioprosthetic aortic valve failure: the valve-in-valve procedure. Circulation. 2013;127(25):2542-2550.

15. Al-Sarraf N, Thalib L, Hughes A, Houlihan M, Tolan M, Young V, McGovern E. Cross-clamp time is an independent predictor of mortality and morbidity in low- and highrisk cardiac patients. Int J Surg. 2011;9(1):104-109.

16. Kosasih M, Almeida AA, Smith JA. Early Outcomes of Sutureless Aortic Valve Replacement With the Perceval S Bioprosthesis. Heart Lung Circ. 2019;28(6):970-976.

17. Nalluri N, Atti V, Munir AB, Karam B, Patel NJ, Kumar $\mathrm{V}$, Vemula $\mathrm{P}$, et al. Valve in valve transcatheter aortic 
valve implantation (ViV-TAVI) versus redo-Surgical aortic valve replacement (redo-SAVR): A systematic review and meta-analysis. J Interv Cardiol. 2018;31(5):661-671.

18. Johnston DR, Soltesz EG, Vakil N, Rajeswaran J, Roselli EE, Sabik JF, 3rd, Smedira NG, et al. Long-term durability of bioprosthetic aortic valves: implications from 12,569 implants. Ann Thorac Surg. 2015;99(4):12391247.

19. Sian K, Li S, Selvakumar D, Mejia R. Early results of the Sorin(R) Perceval S sutureless valve: systematic review and meta-analysis. J Thorac Dis. 2017;9(3):711-724.

20. Powell R, Pelletier MP, Chu MWA, Bouchard D, Melvin $\mathrm{KN}$, Adams C. The perceval sutureless aortic valve: review of outcomes, complications, and future direction. Innovations (Phila). 2017;12(3):155-173.

21. Rodriguez-Gabella T, Voisine P, Puri R, Pibarot P, RodesCabau J. Aortic bioprosthetic valve durability: incidence, mechanisms, predictors, and management of surgical and transcatheter valve degeneration. J Am Coll Cardiol. 2017;70(8):1013-1028. 